\title{
The Transformation of the Heterogeneous Materials under the Fatigue Deformation
}

\author{
S. Kh. Shamirzaev, J. K. Ziyovaddinov, Sh. B. Karimov
}

Physical Technical Institute of Uzbek Academy of Sciences, Tashkent, Republic of Uzbekistan.

Email: jahongirziyo@mail.ru

Received February $24^{\text {th }}, 2012$; revised March $24^{\text {th }}, 2012$; accepted April $23^{\text {th }}, 2012$

\begin{abstract}
The objects of our paper are aluminum alloy samples (AASs) contained the different amount of $\mathrm{Cu}, \mathrm{Mn}, \mathrm{Mg}, \mathrm{Si}$ and $\mathrm{Li}$. We are modeling the features of microstructure of potential relief of an AAS and studying its transformation under both imposed fatigue deformation and wetted by liquid metals ( $\mathrm{Ga}$; or $\mathrm{Hg}$; $\mathrm{Li}$; In). We illustrate the main ideas by using only the "time series" allied with effective internal friction $Q_{e f f}^{-1}$ of an AAS. AASs like B-95 or 7075 are heterogeneous materials for which the more energy can be absorbed by selected micro-regions of a tested sample. So micro-crack in the space of AAS and alarm state of AAS arises. Each micro-region will to contribute the $Q_{k}^{-1}$ (the internal friction $Q_{k}^{-1}$ belong to k-th micro-region) to the effective internal friction- $Q_{e f f}^{-1}$ accordance with fit statistic $g_{k}$. We find a number of micro-regions $-L$ and series $g_{k} \& Q_{k}$ from the experimental data like as the internal friction $\left(Q^{-1}\right)_{\text {eff }}$ versus both the number of cycles $-N$ and the deformation-e. Series $g_{k} \& Q_{k}(k=1,2,3, \cdots, L)$ present the microstructures of AASs. In this paper also is presented the original technology to forecast fatigue damage of an AAS. Here the fatigue sensitive element (FSE) used. We made multiphase heterogeneous mixtures (MHMs) which contents a variable volume of initial components. It is selected MHMs are using for produce FSEs. The present paper is aimed to establish the correlation of the FSEs microstructures changes and corresponding changes of the aluminum alloy microstructures at imposing the same spectra deformation on both of them. A change of FSEs microstructure investigated by using their effective electrical resistance $R_{\text {eff }}$ data.
\end{abstract}

Keywords: Amplitude-Dependent Internal Friction; Fatigue; Cracking Process; Alarm State; Fatigue Sensitive Element

\section{Introduction}

The microstructure of an AAS is reflected on the microstructure of its potential relief that is originated by both a residual deformation and an imposed fatigue deformation. In local regions of polycrystalline alloy undergoes fatigue loads, a wide spectrum of strongly exited states arises $[1,2]$. These states can not be described by traditional methods of a perturbation theory. With the help of mechanics methods of damage one can study only the growth of macroscopic cracks using empirical-formula dependencies describing the growth of a crack.

The various impurities in Aluminum implemented the various resistances to fatigue of an AASs. There is not a common low permits one in advance to determine both what kind and haw much of microstructures will be simultaneously belong to AAS after a number cycles of loads.

The main objective of this paper is to present the experimental method for estimating the effective output parameters of AASs and their change under both the im- posed fatigue deformation and the wetted by liquid metals. The output data of AASs depend on the microstructures they are possessed of. For example, effective internal friction- $Q_{e f f}^{-1}$ of the AAS is formed as a sum of microstructures' internal friction $-Q_{k}^{-1}$ with fit statistics $-g_{k}:$

$$
Q_{e f f}^{-1}=\sum_{k=1}^{L} g_{k} \times Q_{k}^{-1} ; \sum_{k=1}^{L} g_{k}=1 ; \quad(k=1,2,3, \cdots, L)
$$

Damages accumulated at the initial stage of fatigue process change local parameters of the AASs [2-4]. The micro-structures are irreversibly changed if sufficiently high deformation is imposed on AASs. These processes become more intensive and more intricate under wetted by lick of liquid metals such as Gallium - Ga or Mercury - $\mathrm{Hg}$, in this case the AAS become very brittle and reduce their lifetime to zero [5]. As the number of cycle's increases AASs' microstructures eventually begin to make change. As a result the alarm state of an AAS arises. Monitoring of the AASs' alarm states has to been 
developed. It is one of the main object of this paper.

New experimental techniques of $g_{k}$ and $Q_{k}^{-1}$ were developed. The series $g_{k} \& Q_{k}^{-1}$ present the microstructures of polycrystalline Aluminum Alloys [4]. The analysis of both new materials' micro-structures as well as existing materials' micro-structures by novel methods was made.

\section{The Ways of Realization}

After imposing a limited number of cycles of fatigue deformation that change the AASs' microstructures, the test cycle of deformation with selected value of amplitude are imposed on the AASs'.

The $Q_{\text {eff }}^{-1}(\varepsilon)$ ( $\varepsilon$-value of imposed deformation) curve, that was automatically recorded within test cycles of deformation, permits one to bring out the forthcoming structures of AAS and its features, that will take place at the near future cycles of imposed deformation. Reiteration of these processes also for $Q_{\text {eff }}^{-1}-\varepsilon\left(Q_{\text {eff }}^{-1}\right.$ effective internal friction of AAS wetted by liquid metals$\mathrm{Hg}, \mathrm{Ga}, \mathrm{In}$ or $\mathrm{Li}$ ) curve gives one the chance to do the monitoring of the AASs microstructures change.

\section{Internal Friction}

Generally on a fatigue $S N$ curve the numbers of cycles $N$ are within $10^{7}$ to $10^{9}$. If an AAS is wetted by liquid gallium- $\mathrm{Ga}, N$ tends to sharp decrease with the advance of cracking process. It is known [5] that a quantity of Ga leads to embitterment of AAS and decrease its durability under Strain because of cracking processes. The study of micro cracks, related with selected points on surface of a test sample, is essential circumstance for understanding the nature of fatigue. Another essential circumstance is the study of sample's internal friction $\left(Q^{-1}(\varepsilon)\right)$. The internal friction relates to a number of structure-dependent phenomena which permit to judge about fine details of the real structure of a condensed hard matter. For some samples $Q^{-1}(\varepsilon)$ curves possess a hysteretic dependence, which after Granato and Lucke [6] can be related with moving and damping of hesitating dislocation segments in the selected local regions of an
AAS.

But there are ample samples of construction alloys' $Q^{-1}(\varepsilon)$ curves, that meet difficulties under the quantitative comparison with the theory of dislocation damping. This theory was developed for the homogeneous media. The Aluminum Alloys are structurally heterogeneous. So there are overstrained micro regions with high speed evolution of damage processes. Those micro regions are transformed under the load and verged towards to unstable state. They store mechanical energy due to both the phonon mechanism [7] and a structural change which one can notice before micro-cracking processes.

\section{Experimental Installation}

The two-component oscillator (sample and piezoceramics), having an Eigen-frequency of about $18 \mathrm{kHz}$, was used by Mason [8] for study the amplitude dependent decrement on the vibration Strain amplitude. High level of computer engineering was used by Mills D. and Bratina W. J, [9], Kardashov B.K. et al. [10], Schenck H. et al [11] for studying the effect of the oscillation amplitude and temperature on the amplitude-dependent internal friction $Q^{-1}(\varepsilon)$. We also are using the two-component oscillator (ZrTiPb-19 piezoceramics and the wetted by liquid $\mathrm{Ga}$ on the AAS) having an Eigen-frequency of about $20 \mathrm{kHz}$, for studying the effect of the oscillation amplitude and cracking processes on the amplitude-dependent internal friction $Q^{-1}(\varepsilon)$ (see Figure 1).

The fatigue element $(\mathrm{FE})$ is small weight (no more than $5 \mathrm{~g}$ ) and has film geometry. They are produced by thermal vacuum evaporation of the charge onto the polyamide support. The charge consists of a finely dispersed mixture of various initial components, e.g., a mixture of granular $\mathrm{Bi}_{2-\mathrm{x}} \mathrm{Te}_{3+\mathrm{x}}$ and $\mathrm{Sb}_{2-\mathrm{y}} \mathrm{Te}_{3+\mathrm{y}}$ with carbonyl iron [12].

An ample number of grains do not take part in fatigue process of both an AAS and a FE. In parallel with growth of the number of strong deformed grains in AAS as well as in FE, there are also the ample number of grains possessing their original state. The simple model of selected FE describes these situations are given in $[1,2]$. Here is

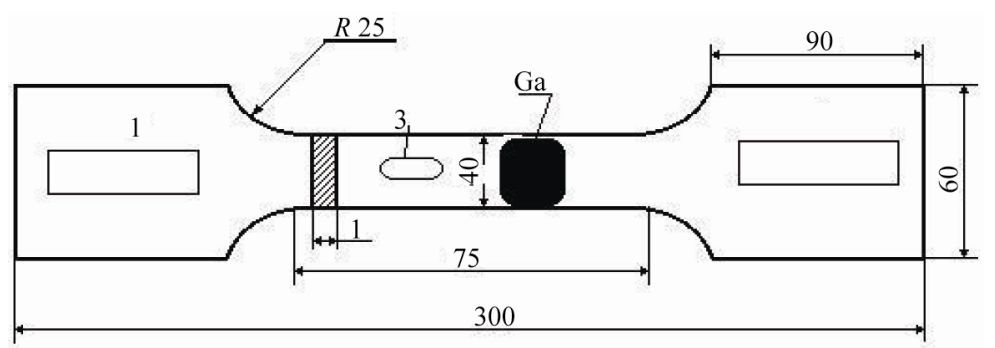

Figure 1. Shape and geometrical dimensions of the Aluminum Alloy Sample. A drop of liquid Ga was imposed on this pattern. After 12 hours this drop became a slur, as pictured at the Figure 1. (1) ZrTiPb-19—oscillator; (2) ZrTiPb-19—receiver; (3) fatigue element of the fatigue sensor. 
also next sufficient conclusion: For all kinds of imposed deformation (extension-compression; bending variations with different coefficients of asymmetry; various spectra of imposed deformations), at which the output parameters (electric resistance $R_{n}$ for FE and internal friction $Q_{n}^{-1}$ for AAS) of materials are recorded in equal time $(\tau)$ intervals, the next recurrent relations take place:

$$
\begin{aligned}
& R_{n+1}[\sigma]=O \times R_{n}[\sigma]=B_{n} \times R_{n}+\left(1-B_{n}\right) \times M_{n} \\
& Q_{n+1}^{-1}[\sigma]=O_{n} \times Q_{n}^{-1}[\sigma]=b \times Q_{n}^{-1}+\left(1-b_{n}\right) \times m_{n}
\end{aligned}
$$

Here: $R_{n}$ is the electric resistance of FE measured under the same state of the environment; $n$ is the ordinal number of measurement $(n=1,2,3, \cdots, g)$; $g$ is the maximum number of the measurement performed; $O_{n}$ is the loading operator transferring the FE resistance from $R_{n}$-th state to $R_{n+1}$-th state; $\sigma$ is a complicated parameter to characterize the type of imposed deformation; the "time series" is composed of experimental data of the types:

$$
\begin{aligned}
& R_{1}, R_{2}, \cdots, R_{n-1}, R_{n}, R_{n+1}, \cdots, R_{q} ; \\
& \left(Q_{1}^{-1}\right)_{e f f},\left(Q_{2}^{-1}\right)_{e f f}, \cdots,\left(Q_{n-1}^{-1}\right)_{e f f},\left(Q_{n}^{-1}\right)_{e f f}, \\
& \left(Q_{n+1}^{-1}\right)_{e f f}, \cdots,\left(Q_{q}^{-1}\right)_{e f f}
\end{aligned}
$$

After imposing a limit number of cycles of fatigue deformation, that changes both the AA's structure and the FE's structure, the test cycles of deformation with selected value of amplitudes are imposed on the sample. The $Q^{-1} \varepsilon$ ( $Q^{-1}$-internal friction of AAS and $\varepsilon-$ value of imposed deformation) curve, that was automatically recorded within test cycles of deformation, permits one to bring out the forthcoming structures of AAS and its features, that will take place at the near future cycles of imposed deformation. Reiteration of these processes gives one the chance to do the monitoring of fatigue features of different Aluminum Alloys Samples as well as of FEs (See [4]).

\section{The General Model}

To gain a greater insight into why the $Q^{-1}(\varepsilon)$ dependencies have the form as in Figure 2 (or Figure 3, before B point), assume that for every overstrained micro-regions the next

$$
Q_{k}(\varepsilon)= \begin{cases}G(m) \cdot \varepsilon & \text { if } \varepsilon \leq \varepsilon_{k} \\ G(m) \cdot \varepsilon_{k} & \text { if } \varepsilon \geq \varepsilon_{k}\end{cases}
$$

formulae takes place; $\varepsilon_{k}$ varies in value. A scale factor $-G(m)$ is the same for each micro-region of the selected AAS and depends on the degree of asymmetry$m$ of the imposed cyclic deformation.

Each micro-region will be able to contribute to the $\Delta Q_{\text {eff }}^{-1}$ accordance with statistic $g_{k}$ [2]:

$$
Q^{-1}(\varepsilon)=\sum_{k=1}^{L} g_{k} \times Q_{k}^{-1}(\varepsilon) ; \sum_{k=1}^{L} g_{k}=1 \text {. }
$$

$Q_{k}^{-1}(\varepsilon, m, N)$-Variation of internal friction of a $k$-th micro-region depends on the amplitude- $\varepsilon$ and degree of asymmetry- $m$ of the imposed cyclic deformation. $N$ is a number of cycles; $L$-number of micro-regions. For the sake of convenience, assume that $Q_{\max }^{-1}=M_{g}(\mathrm{~m})$ and introduce the next value $Z_{k}$ :

$$
Z_{k}=G(m) \times \varepsilon_{k} / M_{g}(m) ; \quad(k=1,2,3, \cdots, L)
$$

$Z_{k}$ and $g_{k}$ can be found from experimental data, Figure 2 (or Figure 3, before the B point), and formulae (4). Proceed as follows:

$$
\begin{aligned}
& Q^{-1}(\varepsilon)=G \cdot \varepsilon \cdot \sum_{k=1}^{L} g_{k}=G \cdot \varepsilon \\
& \text { if } 0 \leq \varepsilon \leq \varepsilon_{1} \\
& Q^{-1}(\varepsilon)=G \cdot \varepsilon_{1} \cdot g_{1}+G \cdot \varepsilon \cdot\left\{1-g_{1}\right\} \\
& \text { if } \varepsilon_{1} \leq \varepsilon \leq \varepsilon_{2} \\
& Q^{-1}(\varepsilon)=G \cdot \varepsilon_{1} \cdot g_{1}+G \cdot \varepsilon_{2} \cdot g_{2}+G \cdot \varepsilon \cdot\left\{1-g_{1}-g_{2}\right\}
\end{aligned}
$$$$
\text { if } \varepsilon_{2} \leq \varepsilon \leq \varepsilon_{3} \text {, and so on. }
$$

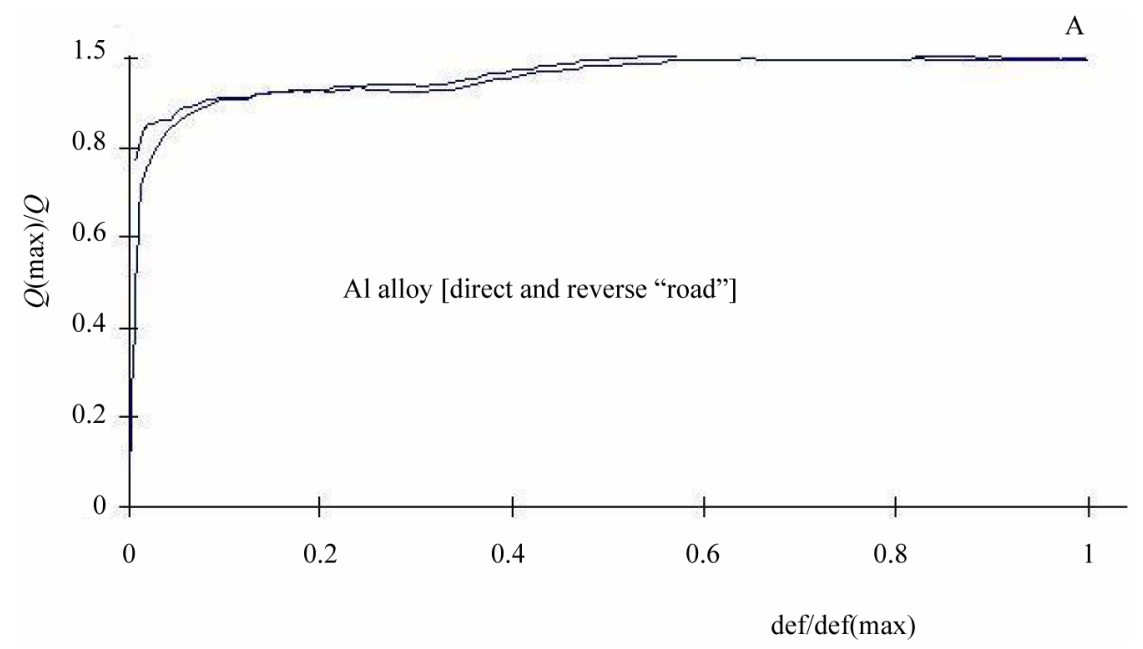

Figure 2. Relative internal friction of Aluminum Alloy Sample (B-95) versus the relative imposed deformation. 
Notice that $\varepsilon_{1}<\varepsilon_{2}<\cdots<\varepsilon_{L}$ and $\varepsilon$ vary in each of interval of $\left[\varepsilon_{k}, \varepsilon_{k+1}\right]$. Let us take a derivative of $Q^{-1}(\varepsilon)$ with respect to $\varepsilon$ in each of Equation (6) equalities. Then each subsequent equality can be subtracted from the preceding one. As a result one finds all quantities of $g_{k}$, $\varepsilon_{k}$, as well as $L ; G(m)$ and $M_{g}(m)$. Figure 4 presents the states of AAS after various numbers of cycles and value of the imposed deformation. Information entropy $S$ :

$$
S=-\sum_{k=1}^{L} g_{k} \times \operatorname{Ln}\left(g_{k}\right)
$$

It is measure of the amount of disorder (different states) in AAS states. This model also admits control of the structural mutations in AAS and FE after $\mathrm{N}$ cycles of an imposed regular deformation [4].

The same procedure was used on to the $Q^{-1}-\varepsilon$ (or $Q^{-1}-N, Q^{-1}$-wetted by liquid gallium-Ga internal friction of AASs) dependencies. Information entropy for this situation $S=1.374$. As a result, one can in advance to bring out the forthcoming structures of FE\&AAS and its features that will take place at the near future cycles of imposed deformation.

High level of computer engineering was used by us to study the effect of the cracking processes, caused by liquid metals, on the amplitude-dependent internal friction $Q^{-1}(\varepsilon)$. The rate of sample mechanical irregularity was found to be different for the different time after wetted by liquid $\mathrm{Ga}$.

In Figures 5 and $\mathbf{6}$ are presented the internal friction $Q^{-1}$ of Aluminum Alloy Samples versus of both the $N$ -cycles and the $\varepsilon$-deformations imposed on AASs (D16-T). At the Figure 7 and Figure 8 are presented the $R_{\text {eff }}$ changes under simple and complicated mode of loads.

\section{The Original Technology to Forecast Fatigue Damage of an Aluminum Alloy}

We shall consider both the fatigue curve of AA (Figure 9) and the equal change resistance curve $(\langle E\rangle-n$ curve for selected values of $\left.r=\left(R_{n}-R_{0}\right) / R_{0}\right)$ of SEs (Figure

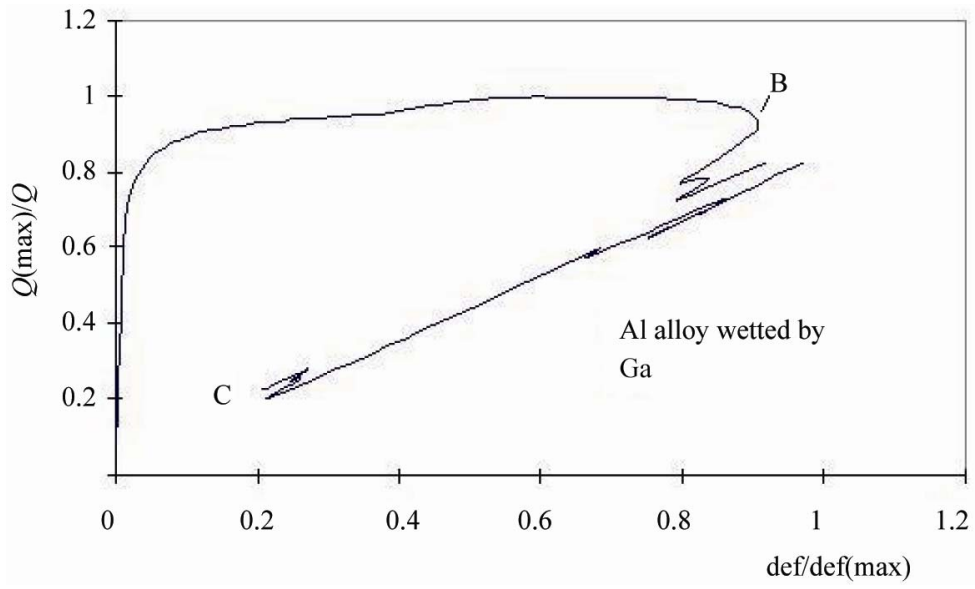

Figure 3. Relative internal friction of Aluminum Alloy's Sample (B-95) wetted by liquid gallium-Ga, versus the relative imposed deformation. This curve reflected the cracking process, beginning from B point. At the $C$ point sample fractured.

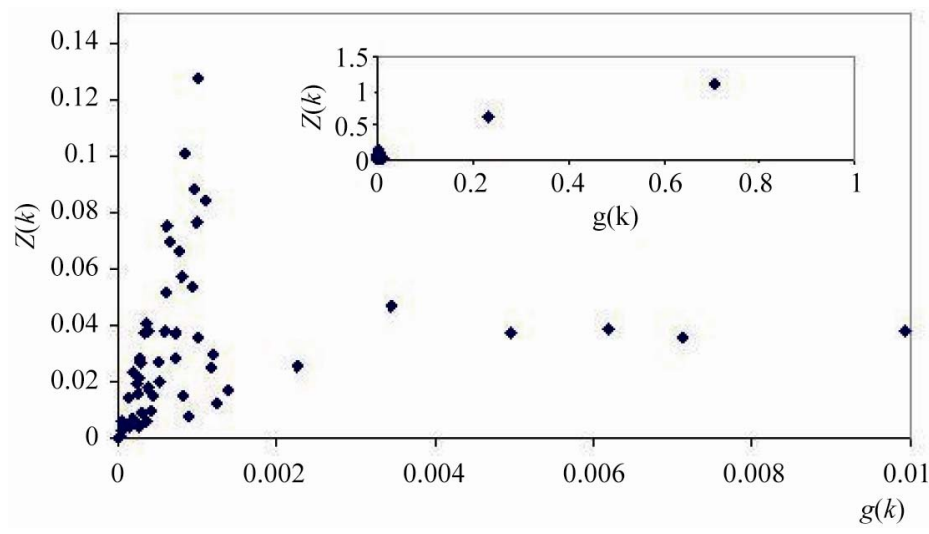

Figure 4. The state $\left(g \& Z_{k}\right)$ of Aluminum Alloy in the end (see point A on Figure 2) of the direct "road". Information entropy for this state $S=\mathbf{0 . 9 5 0}$. The full diagram $\left(g \& Z_{k}\right)$ is inserted the greasy zero points of which are presented on the main figure. 


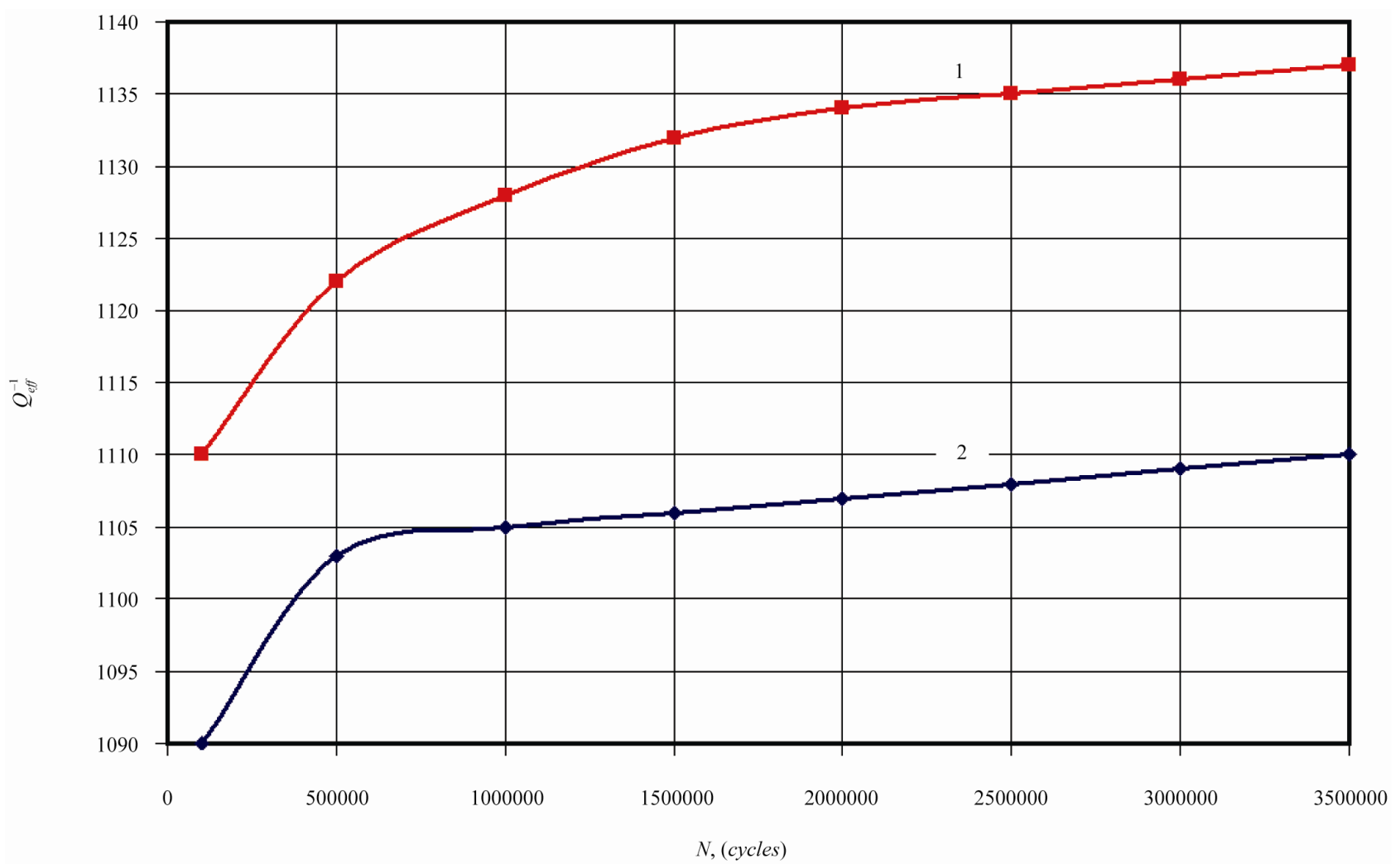

Figure 5. Internal friction $Q^{-1}$ of Aluminum Alloy's Sample (D16-T) versus $N$ cycles of the imposed deformation coresponding both $32 \mathrm{~kg} / \mathrm{mm}^{2}(1)$ and $26 \mathrm{~kg} / \mathrm{mm}^{2}(2)$ loads. The asymmetry coefficient is unity $(m=1)$.

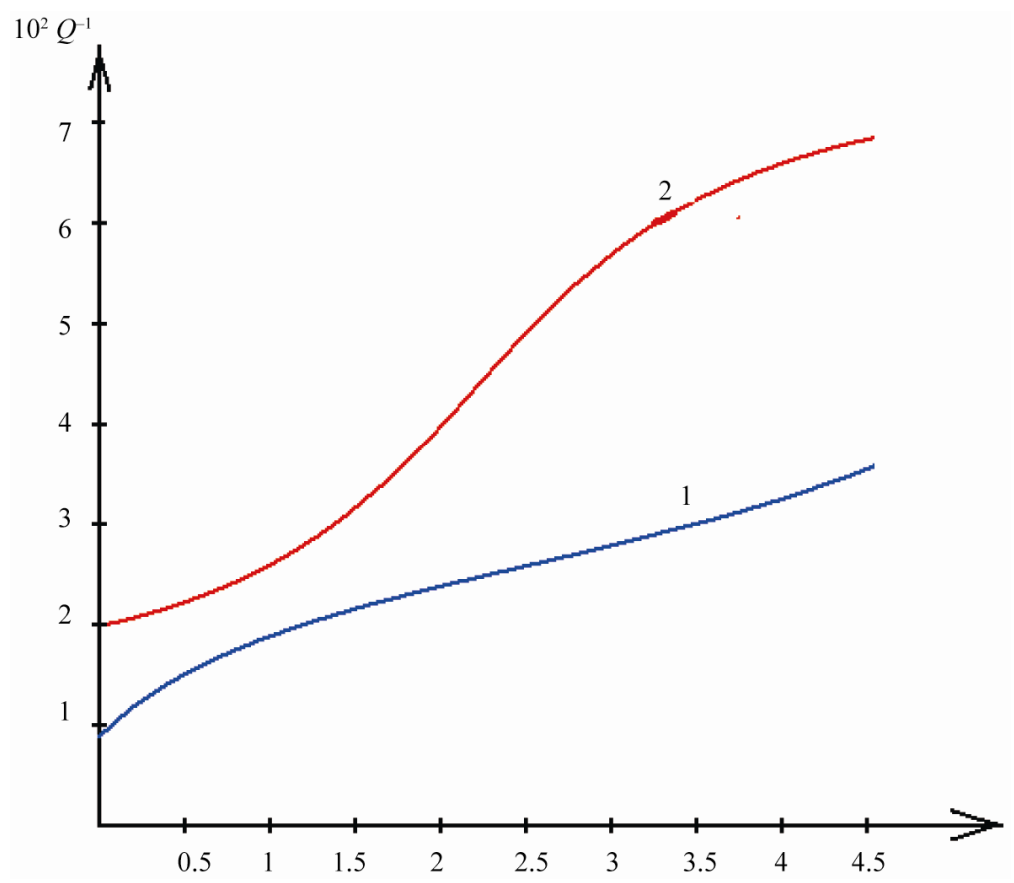

Figure 6. Internal friction $Q^{-1}$ of Aluminum Alloy's Sample (D16-T) versus imposed deformation. (1) After imposed $5 \times 10^{4}$ cycles of $25 \mathrm{~kg} / \mathrm{mm}^{2}$ load; (2) After imposed $5 \times 10^{6}$ cycles of $25 \mathrm{~kg} / \mathrm{mm}^{2} \mathrm{load}$.

10) were obtained at the same conditions of imposed spectrum of deformation.
Our description is very close to one, which was given in [13]. The difference is in the next. Instead of to select 


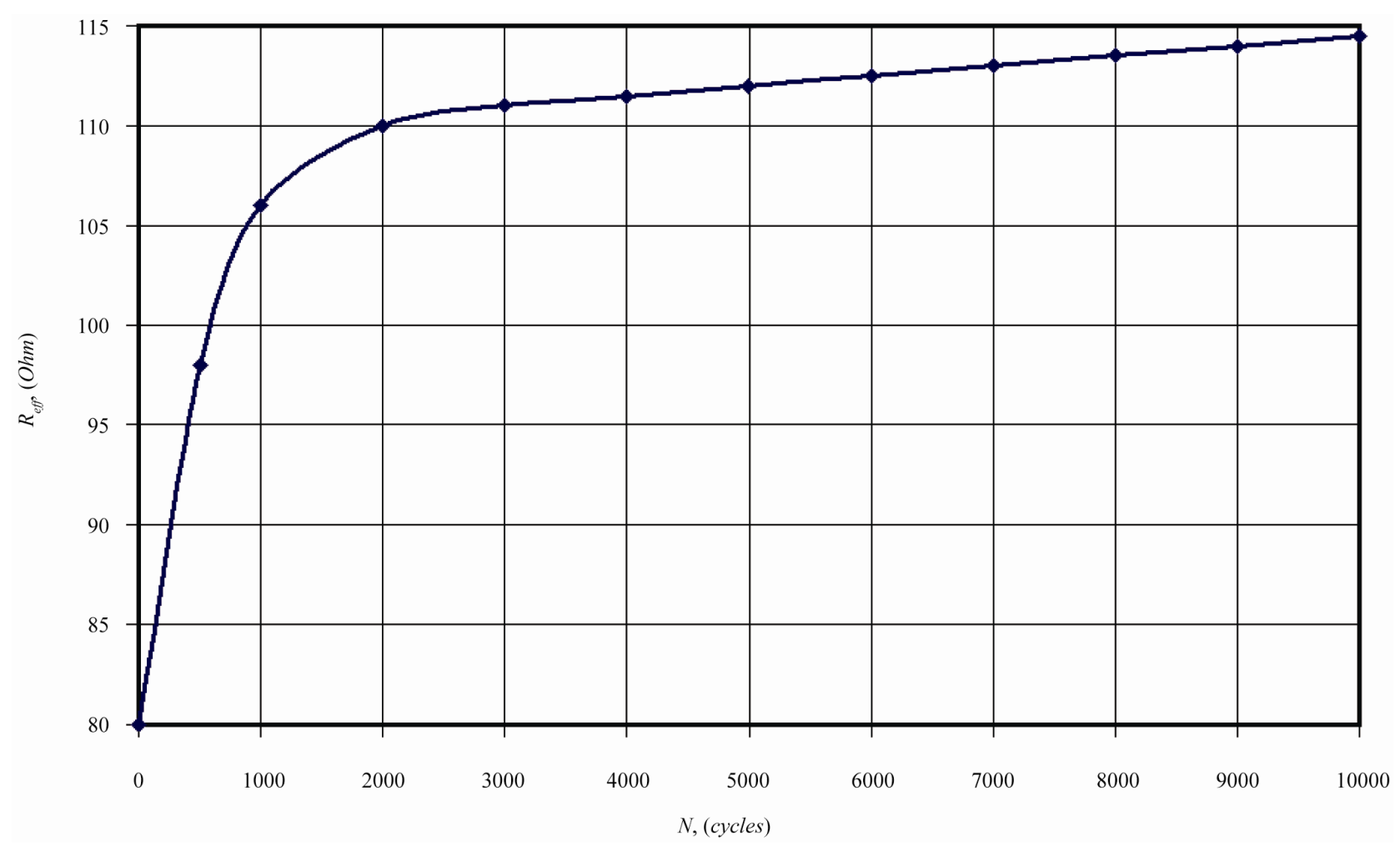

Figure 7. $\boldsymbol{R}_{\text {eff }}$ of SE versus $N$ (sample mode of loads).

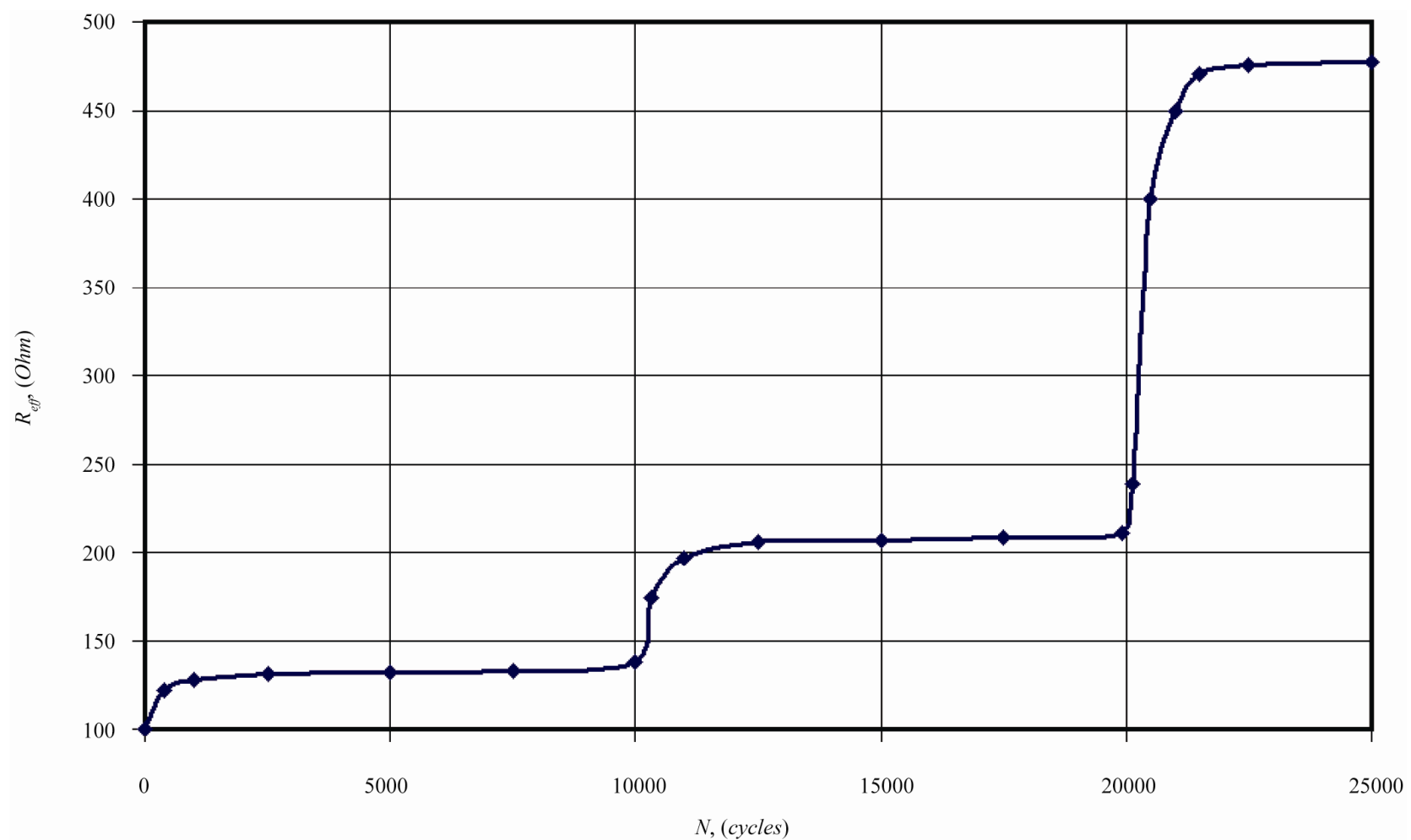

Figure 8. $\boldsymbol{R}_{\text {eff }}$ of SE versus $N$ (complicated mode of loads).

the SE's which ECRC is the "same" as investigated metal, we introduce the alarm state of an Aluminum Al- loy Sample (AAS), it gives us the opportunity to escape the analysis of mechanical amplifier [13]. 


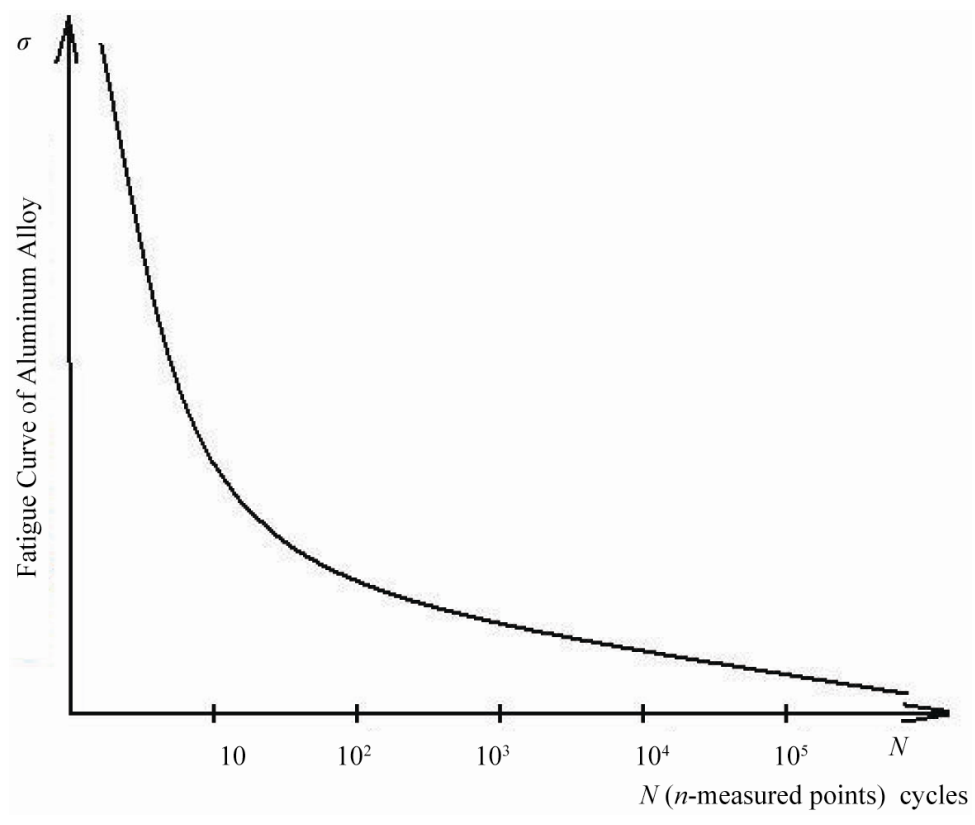

Figure 9. Fatigue curve of an Aluminum Alloy (D16-T) sample.

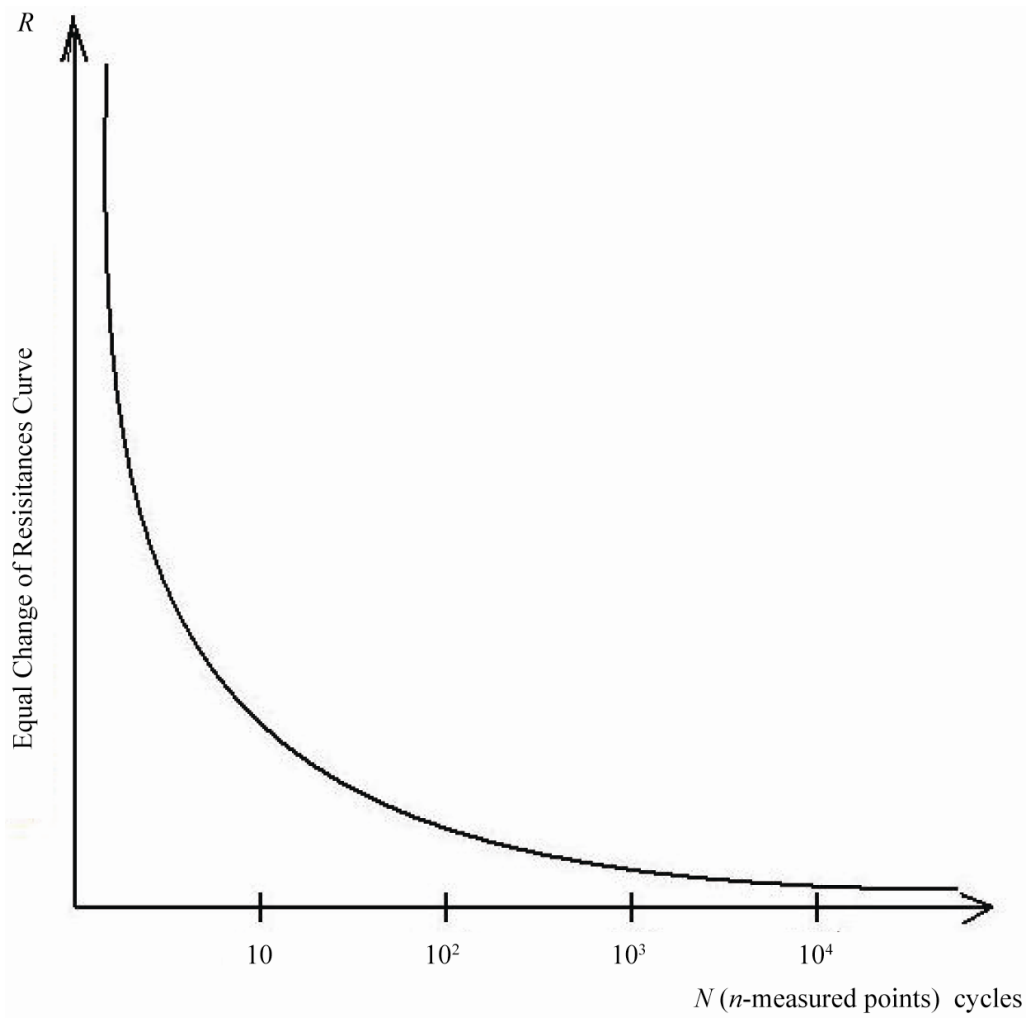

Figure 10. Equal change of resistance curve for SE, rigidly installed on the sample of Aluminum Alloy.

Figure 10 shows the equal change of resistance curves (ECRCs) of FEs rigidly installed at the sample of AA. The rate of spectrum of deformation is the value $\langle E\rangle$ :

$$
\langle E\rangle=\left(\left\langle e_{\phi}^{e}\right\rangle\right)^{1 / 2} \quad\left\langle e_{\phi}^{e}\right\rangle=(1 / \phi) \int_{0}^{\phi} \times r^{\prime} e^{2}(t) \times \mathrm{d} t
$$

$e(t)$-value of imposed deformation; $t$-time; $\phi$-equal time interval at which the resistance's $R_{n}$ were measured. Figure 11 shows $R_{\text {eff }}$ versus $n$ for difference value of $\langle E\rangle$. From these curves one can compose the Figure 12.

Figure 13 shows the operative curve for an AAS and 


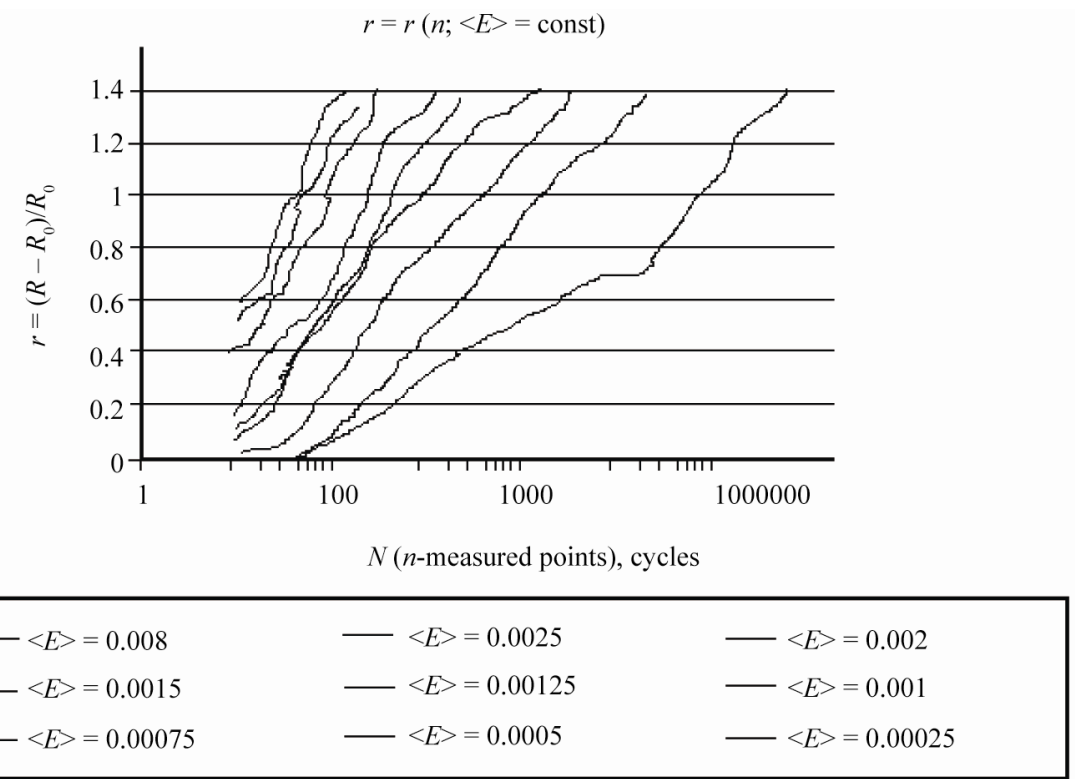

Figure 11. Sensitive elements $r$-n dependence for different value of $<E>$.

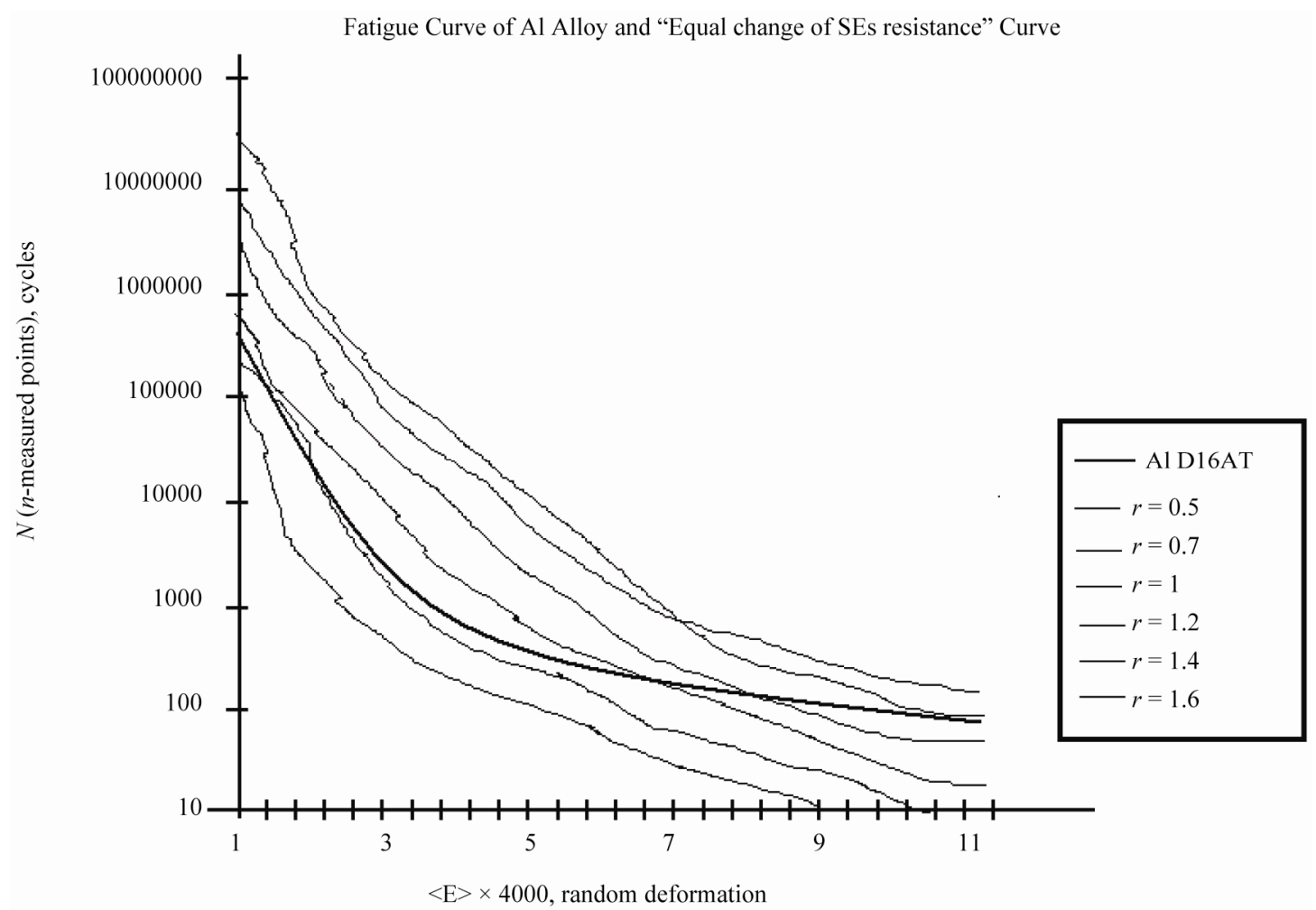

Figure 12. Crossing the Fatigue Curve with equal change of resistance curves forms the Alarm Curve for Aluminum Alloy's Sample.

to be suit for it the Alarm curve. A crossing point of these curves is the service AAS failure. One can adaptive forecast this point using the series Equation (2) and formula Equation (1) [14].

Just bellow we present the methodical example (Me) helping to understand the main idea. (See Figure 14, or
Figure $1 \mathrm{Me} \div$ Figure $4 \mathrm{Me}$ ). At the Figure $1 \mathrm{Me}$ there are two operative curve, which, for the simplicity, made as linear. The first have energy equal E1, the second have energy E2 $(E 2>E 1)$. The second Figure $2 \mathrm{Me}$ is the same as Figure $1 \mathrm{Me}$. But instead of arbitrary fixed second line there one have a failure line. At the Figure $3 \mathrm{Me}$ 


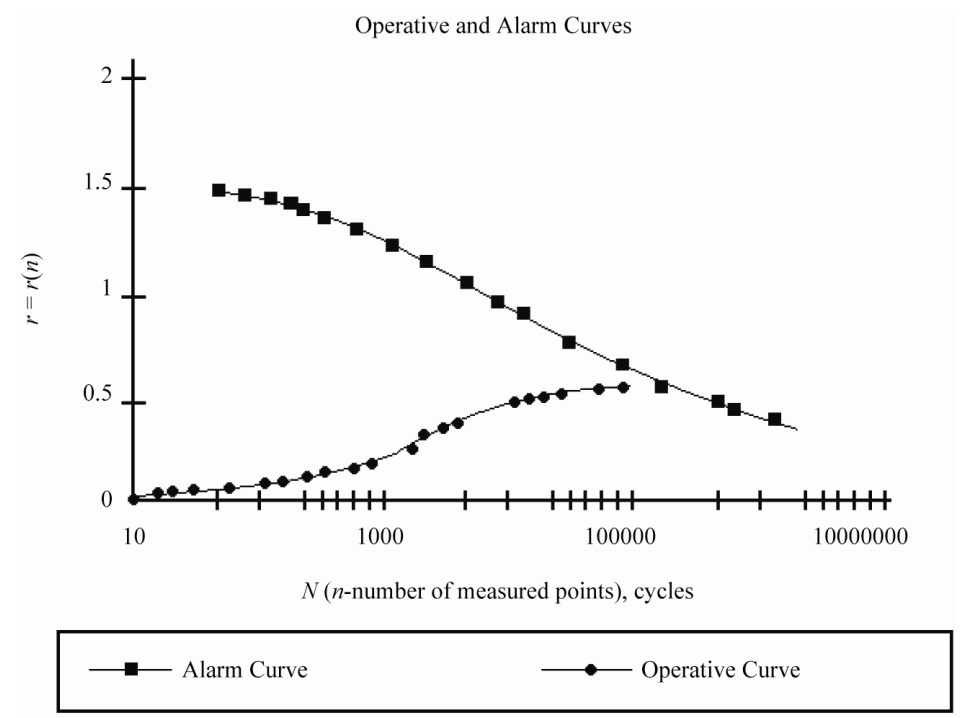

Figure 13. Alarm curve for Aluminum Alloy's Sample and operative curve for sensitive elements, rigidly installed onto this Aluminum Alloy's Sample.

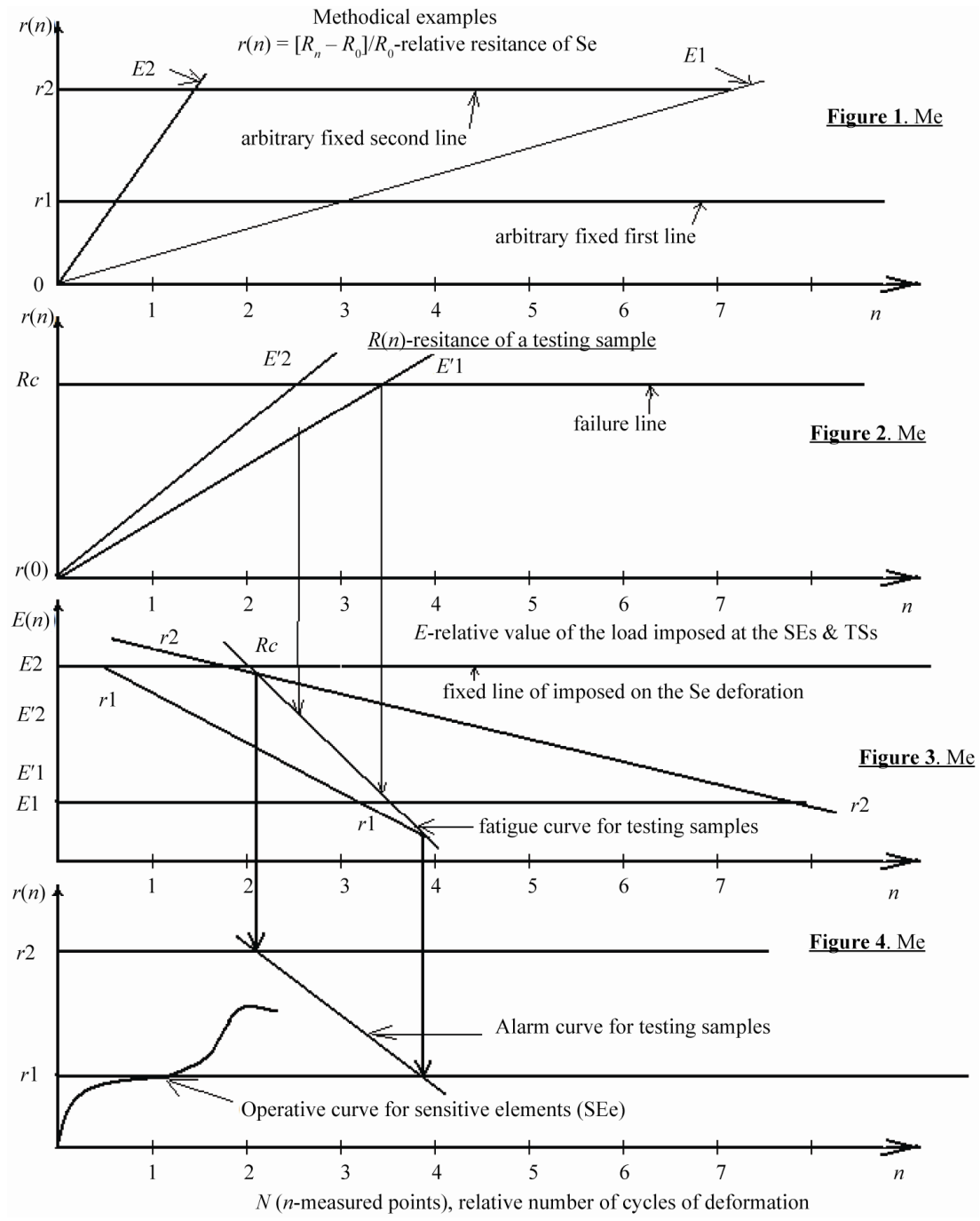

Figure 14. Methodical examples. 
one can see fatigue curve for testing samples and a way haw it may be getting from Figure $4 \mathrm{Me}$. On the Figure $4 \mathrm{Me}$, one can see haw to get the Alarm curve for testing sample, using the data of Figure $4 \mathrm{Me}$ and arbitrary fixed first and second line from Figure $1 \mathrm{Me}$.

\section{REFERENCES}

[1] S. Shamirzaev, "Modelling a Fatigue Imperfection of Structural Materials," International Journal of Fatigue, Vol. 24, No. 7, 2002, pp. 777-782. doi:10.1016/S0142-1123(01)00192-X

[2] S. Shamirzaev, "Response of Mhfms at a Fast and a Slow Operational Loads," A Collection of Technical Papers of the AIAA/ASME/ASCE/AHS/ASC Structures, Structural Dynamics, and Materials Conference and Exhibit, St. Louis, 12-15 April 1999, pp. 1717-1726.

[3] S. Shamirzaev and G. Shamirzaeva, "The Rheological Model of Fatigue Damage of Cm," Proceedings of the 13th International Congress on Rheology, Cambridge, 20-25 August 2000, pp. 377-379.

[4] S. Shamirzaev, S. Ganihanov and G. Shamirzaeva, "The Monitoring of Fatigue Features of $\mathrm{Cm}$ for a Very High Cycle of Loads," Proceedings of the International Conference on Fatigue in the Very High Cycle Regime, Vienna, 2-4 July 2001, pp. 245-252.

[5] W. Rostoker, J. M. McCaughey and H. Markus, "Embrittlement by Liqouid Metals," Reinhold Publishing Corporation, New York, 1960.

[6] A. V. Granato and K. Lucke, "Theory of Mechanical Damping Due to Dislocations," Journal of Applied Phys- ics, Vol. 27, 1956, pp. 583-593. doi:10.1063/1.1722436

[7] V. S. Kuksenko, V. I. Betechtin, V. S. Ryskin and A. I. Slutsker, "Nucleation of Submicroscopic Cracks in Stressed Solids," International Journal of Fatigue, 1975, Vol. 11, No. 5, 1975, pp. 829-840. doi:10.1007/BF00012900

[8] W. P Mason, "Physical Acoustics and the Properties of Solids," Journal of the Acoustical Society of America, Vol. 28, No. 6, 1956, pp. 1197-1219. doi:10.1121/1.1908593

[9] W. J. Bratina, "Physical Acoustics," Academic Press, New York, 1966.

[10] B. K. Kardashev, S. P. Nikanorov and O. A. Voinova, "Amplitude Dependent Internal Friction in NaF Crystals in the Temperature Range 295 to $4.2 \mathrm{~K}$," Physica Status Solidi (a), Vol. 12, No. 2, 1972, pp. 375-379. doi:10.1002/pssa.2210120204

[11] H. Schenck, E. Schmidtmann and H. Kettler, "Effect of Strain Aging on Processes Occurring in Cyclic Loading of Steel," Arch Eisenhuettenw, Vol. 31, 1960, p. 659.

[12] S. Shamirzaev, "Mhms for Fatigue Gage," Iron and Steel Institute of Japan Proceedings of the 7th International Conference, Steel Rolling '98, Chibo, 9-11 November 1998, pp. 844-849.

[13] V. T. Troshchenko and V. I. Boiko, "Fatigue Damage Sensor and Substatiation of Its Application, Communications 1 and 2," Problemi Prochnosti, Vol. 1, No. 187, 1985, pp. 3-14

[14] S. Shamirzaev, "The Theory of Output Parameters of a Pressing Powder Mixture with Random Packaging Density," Solid State Sciences, Vol. 6, No. 10, 2004, pp. 1125 1129. doi:10.1016/j.solidstatesciences.2004.07.004 